\title{
Philosophiques
}

\section{Le réel en dépit du réalisme}

\section{Michel Bitbol}

Volume 47, numéro 2, automne 2020

URI : https://id.erudit.org/iderudit/1075136ar

DOI : https://doi.org/10.7202/1075136ar

Aller au sommaire du numéro

Éditeur(s)

Société de philosophie du Québec

ISSN

0316-2923 (imprimé)

1492-1391 (numérique)

Découvrir la revue

Citer ce document

Bitbol, M. (2020). Le réel en dépit du réalisme. Philosophiques, 47(2), 471-492.

https://doi.org/10.7202/1075136ar d'utilisation que vous pouvez consulter en ligne.

https://apropos.erudit.org/fr/usagers/politique-dutilisation/ 


\title{
Le réel en dépit du réalisme
}

\author{
MICHEL BITBOL \\ Archives Husserl, CNRS/ENS, 45 rue d'Ulm, Paris
}

La Disputatio, telle que je la comprends, est une œuvre coopérative plutôt que concurrentielle. Elle a beau prendre la forme d'un débat contradictoire entre interlocuteurs soutenant des positions distinctes, elle tend vers un telos qui leur est commun. La succession des raisons, l'alternance des arguments et des contre-arguments jusqu'à épuiser leurs ressources, n'y est pas censée déboucher sur un constat silencieux de désaccord, mais sur une proposition généralement recevable comme vraie. Pour; contre; et détermination finale. Tel est l'ordre ternaire de la Disputatio scolastique ${ }^{1}$. Si l'on veut redonner à ce programme la crédibilité qu'il a perdue en sortant de l'enclos médiéval de consensus impératif, il faut procéder à des aménagements et à des amplifications.

L'amplification centrale consiste à admettre que le débat peut aussi porter sur les questions limites de la philosophie; c'est-à-dire sur les présupposés servant de cadres tacites aux débats philosophiques. Parmi les présupposés, il y a celui qui fixe le genre d'argument recevable. Les arguments philosophiques doivent-ils rester sur le plan de la logique déductive (ce qui suppose non seulement un cadre définitionnel et axiomatique partagé, mais aussi une discipline cognitive unanime) ? Peut-on en outre faire usage d'arguments d'autorité textuelle (comme dans la théologie médiévale), d'arguments de contradiction performative, voire d'arguments ad hominem? Peut-on aller jusqu'à inviter l'adversaire à constater en silence et dans son for intérieur la contradiction à laquelle sa position l'entraîne ${ }^{2}$, alors que cela implique de l'attirer temporairement hors de la sphère du logos? Est-il licite d'indiquer à l'interlocuteur que le discours qu'on lui tient ne se comprend pleinement que sous la condition d'un certain mode d'être, d'une attitude fondamentale (comme l'épochè en phénoménologie, ou l'"attitude naturelle» dans la plupart des autres cas) ${ }^{3}$ ? Ce sont des interrogations de cette famille que soulève Isabelle Thomas-Fogiel lorsqu'elle cherche à savoir comment on peut passer du "je" au "nous" face à "pareille promotion du saisissement muet »; ou lorsqu'elle se demande comment parler "d'un contenu de l'onto/logie, si le type de parole qui y est pertinente ne relève plus du logos ».

1. B. Périgot, «Antécédences: de la disputatio médiévale au débat humaniste», Memini, 11, 43-61, 2007; B. Périgot, Dialectique et littérature. Les avatars de la dispute entre Moyen Âge et Renaissance, Honoré Champion, 2005.

2. Maintenant la finitude, p. 135.

3. M. Bitbol, La conscience a-t-elle une origine?, Flammarion, 2014, Questions 4 et 7.

PHILOSOPHIQUES 47/2 - Automne 2020, p. 471-492 
J'entends cette inquiétude à propos d'un risque de sortie de route extra-philosophique. Indubitablement, les questions portant sur les genres d'arguments recevables, ou sur la dépendance des argumentations vis-à-vis de la posture existentielle des interlocuteurs, invitent à visiter les coulisses de la philosophie, à donner un coup de sonde dans son envers pré-logique. Mais, à mon sens, elles ne sortent pas pour autant de son champ disciplinaire. Elles font partie, à un degré plus élevé que la question «qu'est-ce que la philosophie ?» ${ }^{4}$, du domaine méta-philosophique. Si l'on admet que la philosophie est cette discipline exceptionnelle qui non seulement n'exclut pas ses propres méta-questions, mais s'en nourrit (faute de discipline plus englobante qu'elle), alors les questions limites indiquées plus haut sont pleinement philosophiques.

Reprenons-les en tant que telles. Faire allusion au « saisissement muet » du sujet-philosophe, mettre ouvertement en scène la diversité des états de conscience (naturels ou réflexifs) des interlocuteurs du débat philosophique, cela implique-t-il vraiment de renoncer à atteindre un accord universel? Il me semble au contraire que c'est en ne mettant pas pleinement en lumière la variété des genres d'arguments, et l'incompatibilité de leurs pré-conditions vécues, qu'on risque la fragmentation et l'incompréhension. Car alors, les éléments du débat sont entendus par chacun sous ses propres arrière-plans insus, au lieu d'être rapportés à ceux de son interlocuteur, qui seuls leur donnent sens et pertinence. Supposons à l'inverse qu'on élargisse le débat jusqu'à interroger l'horizon existentiel sur lequel se découpe chaque thèse, et jusqu'à instaurer les conditions discursives d'une réflexion sur les positions pré-discursives des protagonistes. On se hisse alors au niveau d'une reconnaissance mutuelle des points de vue, semblable à celui qu'on aimerait atteindre dans le dialogue des cultures. Faute de pouvoir s'accorder sur une thèse par des raisons, on peut au moins s'entendre sur les raisons de l'irréductibilité des thèses ${ }^{5}$. Un espace de pensée agrandi est ainsi rendu accessible, faisant des raisons du désaccord l'instrument d'un possible méta-accord. En résumé, pour s'approcher de son idéal d'un accord universel sans trop compter sur les ressemblances entre les interlocuteurs, la philosophie a intérêt à élargir ses procédés à la prise en considération raisonnée de leurs plus fortes dissemblances.

C'est dans cet esprit que j'aborde la présente «dispute». En espérant, bien sûr, répondre aux objections de façon satisfaisante. Mais aussi et surtout en recherchant les termes d'un accord, ou au moins d'un méta-accord.

Même avec Quentin Meillassoux, un méta-accord est loin d'être inconcevable par-delà le choc des contre-arguments que je lui ai opposés dans Maintenant la finitude. Le processus de convergence avec Meillassoux ne saurait en aucun cas consister pour moi à adopter une forme de «maté-

4. G. Deleuze et F. Guattari, Qu'est-ce que la philosophie? Éditions de Minuit, 1991.

5. Voir B. Van Fraassen, The Empirical Stance, Yale University Press, 2004. 
rialisme ", contrairement à ce que suggère malicieusement Anna Longo (j’y reviendrai). Mais il faut bien reconnaître de nombreux indices d'une résonance souterraine.

Cette résonance est d'abord personnelle. En discutant deux fois longuement avec Quentin Meillassoux, j’ai été impressionné par sa façon de prendre la pensée au sérieux, d'en incarner les conséquences, d'en faire un enjeu de vie, et d'accueillir pourtant avec respectueuse amitié celui qui lui fait courir le risque de la déstabilisation intellectuelle. J'ai eu ainsi l'impression de partager avec lui l'essentiel, par-delà les oppositions souvent diamétrales.

La résonance est aussi intellectuelle, si l'on admet que les oppositions sont souvent de simples renversements de perspectives. Isabelle ThomasFogiel relève avec précision quelques-unes de ces convergences furtives. Possibilité (si ce n'est effectivité) que le monde soit chaos; dépendance de l'ontologie à l'égard d'une certaine conception de la science; nécessaire contingence du principe ultime; et quête d'une forme d'absolu ${ }^{6}$. Mais, comme le remarque également Isabelle Thomas-Fogiel, chaque convergence entre la position de Meillassoux et la mienne ne s'accomplit qu'en passant de l'autre côté d'un miroir de pensée. Le nœud de l'inversion est simple et systématique: à la percée transitive de Meillassoux répond ma récollection intransitive; à sa traversée de l'expérience en direction de quelque chose, répond ma prescription de se redéposer dans l'expérience ou dans les pratiques, d'y faire retour sans directionnalité.

Reparcourons un à un ces renversements de perspective.

Si j'accepte que le monde pourrait ne pas être ultimement ordonné, ce n'est pas au terme d'une percée spéculative aboutissant à faire du chaos l'ultime «loi» d'un monde "sans nous». C'est par le biais d'un retour réflexif sur notre physique statistique. La physique statistique fait émerger l'ordre thermodynamique de l'hypothèse du désordre microscopique, alliée à celle d'une connaissance imparfaite des distributions désordonnées. La physique statistique fait par exemple émerger l'ordre thermodynamique de l'hypothèse du «chaos moléculaire » de Ludwig Boltzmann, ou d'une norme de "découpage grossier» de l'espace des configurations moléculaires énoncée par Josiah Willard Gibbs. L'ordre thermodynamique est donc dans une large mesure le nôtre. Il est celui d'un être qui s'astreint à n'ajouter qu'un minimum d'information microscopique conjecturale à celle que les mesures macroscopiques (de température, de volume et de pression) lui rendent disponible 7 . Qu'un ordre légal puisse émerger à nos yeux d'un désordre extrême, d'un désordre chaotique, est donc avéré. Mais que ce

6. Maintenant la finitude, chapitre VIII.

7. E.T. Jaynes, «Information Theory and Statistical Mechanics ", cité et commenté dans Maintenant la finitude, p. 255. 
désordre supposé soit la nature ultime du monde est indécidable dans le cadre de la physique statistique.

On s'éloigne encore davantage du matérialisme spéculatif avec la physique quantique. Cette dernière rend en effet la thèse d'un désordre inhérent à la nature d'un monde extérieur-sans-nous plus qu'indécidable: invraisemblable. L'usage des probabilités à un niveau fondamental en théorie quantique, semble, il est vrai, conforter la thèse d'un chaos du monde. Mais à l'examen attentif, on se rend compte que cette forme probabiliste n'est pas imposée par une indétermination propre au monde, pas plus qu'elle ne l'est par notre connaissance incomplète d'un monde déterministe. Elle dérive de l'indivisibilité bohrienne du phénomène microscopique en une part nôtre et une part du monde. Elle découle, en d'autres termes, de l'impossibilité de nous séparer du monde ${ }^{8}$. On est ici aux antipodes du réalisme soustractif de Meillassoux; aux antipodes de son attribution d'hyperchaos au seul monde «sans nous».

Si je fais dépendre l'ontologie d'une réflexion sur les sciences, ce n'est que de manière négative, et à titre de point d'appui problématique pour l'imagination. Cela s'oppose à l'approche directe de Meillassoux, qui tient positivement (fût-ce à titre hypothétique) la description mathématique des objets pour un dévoilement de leur «en soi». Mais en quoi consiste cet enseignement ontologique négatif? Ce que plusieurs sciences ${ }^{9}$ nous indiquent, c'est que notre effort de distanciation vis-à-vis du connu, notre projet de déconvolution de la connaissance en deux pôles archétypaux sujet-objet, ne peut pas être poursuivi jusqu'à son terme. Face à ce «non » cryptique, deux stratégies s'esquissent. La première consiste à ne rien demander de plus, à convertir la non-distanciation en directive d'action. C'est ce que propose de faire le «probabilisme radical» de De Finetti, dont Anna Longo se fait l'avocate. Selon le probabilisme radical, écrit-elle, «on ne doit pas se soucier de l'ordre métaphysique du monde, mais seulement de la probabilité de succès de nos paris (décisions d'action)». La seconde stratégie, que suit un probabiliste pas moins radical comme Christopher Fuchs, consiste à esquisser une raison ontologique de se déprendre des ontologies d'objets. Cette raison ontologique de critiquer les ontologies traditionnelles s'inspire d'une philosophie de l'incarnation; elle s'apparente dès lors à l'endo-ontologie merleaupontienne. Mais, contrairement à Christopher Fuchs, je n'en fais pas un motif suffisant pour adhérer à un "réalisme participatif» (un réalisme de la participation intime de l'être connaissant au monde connu). Je n'accorde à

8. Maintenant la finitude, p. 237-240; M. Bitbol, «L'indéterminisme entre deux infinis: absence de causes ou excès non maitrisable de conditions? », sous la direction de P. Bourgine, D. Chavalarias et C. Cohen-Boulakia, in Déterminismes et complexités: du physique à l'éthique. Autour d'Henri Atlan, Éditions La Découverte, 2008; K. Popper, L'univers irrésolu, Hermann, 1984.

9. Avant tout la physique quantique et les sciences cognitives. 
l'endo-ontologie nulle capacité à découvrir la nature des choses; seulement celle d'éviter un désaccord flagrant entre notre désir d'auto-positionnement figuratif et nos directives d'action.

Si je conclus à la nécessaire contingence du principe ultime, ce n'est pas à l'issue d'un raisonnement, mais d'un saisissement; et cela concerne non pas un principe pensable ou formulable, mais la source pensante et formulante. Ce qui est contingent ici, ce n'est pas le monde extérieur, qui pourrait se voir attribuer en propre une détermination hyperchaotique. C'est le "c'est ainsi» indivis, insoustractible, d'une expérience-située-dumonde (je, ici, maintenant, confronté à ceci). Ce nœud déictique ne saurait se voir attribuer une raison. Il demeure sans raison non pas parce qu'il est irrationnel, mais parce que c'est lui qui pré-conditionne la raison ${ }^{10}$, parce que c'est à partir de lui que se déploie le jeu de mise en ordre rationnel des phénomènes. Quant à ce qui est nécessaire, dans ce cas, ce n'est pas la contingence du monde, mais le Je-ici-maintenant apte à découvrir sa propre contingence dans un aperçu immédiat resserrant l'acte du cogito cartésien sur son propre surgissement. Essayez donc de constater, stupéfait, votre propre contingence (pourquoi moi, ici, maintenant, entouré de tout cela?) sans être-constatant. Contrairement à ce que soutient Anna Longo, ces réflexions offrent bien moins que ce que demande Meillassoux pour soutenir sa thèse d'un monde extérieur nécessairement dénué de raison. Mais, dans la mesure où elles mettent en jeu l'insécabilité de l'expérience-située$d u$-monde, elles ont aussi une portée plus vaste que la thèse de Meillassoux.

Si je me mets en quête d'un absolu, c'est en sachant que ma quête est de trop. Ma quête est de trop parce qu'elle me porte à chercher l'absolu ailleurs que là où je(il) me(se) trouve à l'instant: dans le petit extérieur de l'univers visible, ou dans le grand extérieur de son fondement transcendant; dans l'étendue explorable de l'espace-temps quadridimensionnel, ou dans les replis inexplorables de quelques dimensions supplémentaires postulées ${ }^{11}$; dans une chose à laquelle je me corrèle, ou dans le corrélat lui-même, une fois nommé et chosifié. L'absolu dont je me mets en quête ne se trouvant nulle part là-bas au bout de ma quête, il n'est pas question de le penser. Il n'est pas question de le désigner par un mot ou une périphrase (fût-ce le mot "corrélat»), mais de me reconnaître "transfixié " ${ }^{12}$ par lui, en lui ... Mais sans lui, parce que "lui», ce pronom en troisième personne, est encore trop dire. En écrivant ces phrases allusives, je n'essaye (évidemment) pas de pointer un doigt vers l'absolu, mais de recueillir l'attention des lecteurs sur la prémice absolument présente des gestes de pointer, de chercher, d'enquêter.

10. Maintenant la finitude, p. 141.

11. B. Greene, L'univers élégant, Robert Laffont, 2000.

12. Maintenant la finitude, p. 453. 
S'il fallait récapituler ce jeu de parallèle inversé, je dirais que la « réalité» de Meillassoux est trop étroite à mon goût. Trop étroite? Comment accorder ce jugement avec la motivation et le fruit débordants de la démarche du matérialisme spéculatif? Sa motivation est de faire éclater la sphère close de la subjectivité, du langage, des pratiques, pour nous faire déboucher sur le "grand dehors" infini. Sa motivation est de vaincre la claustrophobie épistémologique de qui découvre la marque de l'activité humaine dans tous les contenus de la connaissance, en remettant enfin en scène «les choses mêmes» au sens le plus autonome et le plus extérieur de l'expression. Son fruit, quant à lui, consiste à admettre qu'à monde réel rien d'impossible. Le monde réel du matérialisme spéculatif a pour loi l'absence de lois valant partout et toujours. Le monde réel du matérialisme spéculatif inclut non seulement les mondes de science-fiction, mais les mondes hors sciences. Le monde réel du matérialisme spéculatif est déclaré capable d'une créativité foisonnante et (presque) sans limite; jusqu'à être gros de Dieu lui-même dans l'indéfini inépuisable de ce qui vient ${ }^{13}$. Par comparaison avec cette «réalité »-là, toutes les réalités conçues jusqu'alors semblent autant de carcans pour la pensée. Pourtant, à cette "réalité" manque presque tout. Il manque en elle tout ce qu'il a fallu en soustraire pour la rendre «extérieure». Il manque en elle rien de moins que "nous"; nous qui avons fait l'effort de nous rendre assez transparents pour pouvoir la poser comme un objet devant nous, c'est-à-dire justement comme un «monde sans nous ».

Mais enfin, protestera-t-on, ce qui semble manquer ici n'est presque rien: une poussière composite de carbone, d'azote, d'oxygène et d'hydrogène sur une petite planète bleue à la périphérie d'une galaxie ordinaire parmi des milliards d'autres galaxies. Et d'ailleurs, cette poussière humaine n'est-elle pas généreusement incluse dans le monde surabondant du matérialiste spéculatif? En tant que corps matériel, bien sûr, mais aussi, conjecturalement, en tant que conscience. Car, une fois le principe de raison suspendu, l'apparition spontanée de la conscience dans ce monde matériel fait partie des possibles ${ }^{14}$, un possible aussi improblable mais aussi décisif que celui du Dieu à venir. Poussière terrestre ou éclat sensible contingent à la surface de la matière obtuse. Partie ou moment de la chose matérielle. Telle est la seule place réservée par le matérialisme spéculatif à ce que nous avons pourtant identifié comme l'origine nécessaire de tout jugement, y compris du jugement sur la contingence de l'origine.

Le matérialisme spéculatif rejoint ici le rêve naturaliste de la science moderne, cueilli en peu de mots par Merleau-Ponty: "Alors le monde se fermera sur lui-même et [...] nous serons devenus parties ou moments du

13. Q. Meillassoux, «Deuil à venir, Dieu à venir», Critique, 704-705, 105-115, 2006.

14. Q. Meillassoux, «Iteration, Reiteration, Repetition, a Speculative Analysis of the Meaningless Sign », Conférence du 20 avril 2012, Freie Universität, Berlin. 
Grand Objet ${ }^{15}$.» Le naturalisme prétend ne pas «nous» avoir oubliés: ne nous situe-t-il pas quelque part dans la nature, à titre de fragment du Grand Objet auquel il assimile cette nature? Mais Merleau-Ponty, en bon phénoménologue, pointe l'inconsistance d'un tel idéal au centre même de son énoncé. Sa phrase complète est: "Alors le monde se fermera sur lui-même et, sauf par ce qui, en nous, pense, et fait la science, par ce spectateur impartial qui nous habite, nous serons devenus parties ou moments du Grand Objet. » Cette incidente de la phrase de Merleau-Ponty a été intentionnellement omise dans la première occurrence de la citation. Aveuglée dans la phrase citée, elle fait ressortir le point aveugle du naturalisme, le point que le naturalisme ne veut ou ne peut pas voir parce qu'il s'agit du voyant. La science de la nature oublie "ce qui, en nous, pense et fait la science "; elle l'oublie parce qu'à force de penser et de faire, elle ne prend garde ni à la pensée, ni au faire, ni à la poussée inchoative du pensant et du faisant.

Mais tout de même, répliquera-t-on encore, cet oubli qu'on vient de relever n'est même pas l'oubli de presque rien; il est l'oubli de vraiment rien. Le nous pensant et faisant la science n'est qu'un sujet transcendantal vide, une condition inaperçue de l'apercevoir, un non-objet, un non-étant. On sait que pour Merleau-Ponty il en va tout autrement. Ce qui a été rejeté selon lui dans les arrière-gardes oubliées de l'avancée scientifique, est toute la plénitude incarnée de la vie vécue, dont l'acte de connaître n'est que le fer de lance. C'est ici qu'il faut faire droit aux réflexions pertinentes de Florian Forestier, qui donnent chair à mes caractérisations schématiques de l'arrièreplan connaissant de la connaissance. Il faut faire droit à l'«épaisseur» de l'expérience du connaissant, à toutes ses composantes latentes, périphériques, atmosphériques, émotionnelles, cénesthésiques. Comme l'écrit Florian Forestier, «mon être au monde est coloré, brouillé, intensifié par ces expériences toujours singulières que sont la fatigue, l'épuisement, la distraction, la rêverie. [...] On peut résumer ces considérations par le terme d'incarnation ". Dès lors qu'on n'ignore pas cette épaisseur vécue et charnelle qui nous constitue de part en part, ce qu'il faut soustraire de nous pour atteindre une connaissance objective s'avère immense, excessif, « démesuré». «Objectiver, se mettre en quête du réel, n'est pas seulement se rendre attentif et réceptif à lui, mais toujours aussi creuser, sculpter, modeler l'expérience", poursuit Florian Forestier. La «réalité objective» est produite par une opération qui s'apparente à la sculpture, c'est-à-dire à l'extraction de masses colossales hors du bloc de l'expérience pour en dégager une forme svelte intersubjectivement reconnaissable. Tout ce qui est ressenti, tout ce qui résonne, tout ce qui s'émeut, tout ce qui relève des tonalités affectives, tout ce qui s'inscrit dans des circonstances uniques ou dans des relations interpersonnelles sans précédent, est enlevé du marbre de l'expérience pour laisser affleurer les linéaments reproductibles d'un «monde sans nous ». Il en résulte que l'expé-

15. M. Merleau-Ponty, Le visible et l'invisible, Gallimard, 1964, p. 31. 
rience, notre expérience, est bien plus dense que le résidu objectivé que nous appelons «monde extérieur». Elle contient de quoi constituer la totalité de ce que nous rangeons sous le nom de "réalité extérieure", y compris ses manifestations observables, y compris son concept d'animal vertical rampant sur le sol d'une planète bleue, y compris le foisonnement (pensé) de ses possibles, y compris ses structures mathématiques, y compris les nombres transfinis et l'«infini absolu» du mathématicien Cantor. Mais l'expérience, notre expérience, enveloppe de surcroît l'«épaisseur» incarnée que fait valoir Florian Forestier, cette épaisseur au regard de laquelle l'armature du monde objectivé n'est qu'une toile arachnéenne ténue. Ce qui manque dans le «monde sans nous» du matérialiste spéculatif, ce qui a été soustrait pour l'obtenir, est donc bien presque tout. La pulpe du réel excède de très loin la «réalité extérieure». Le réel s'auto-révèle pleinement en dépit du réalisme.

Les phrases qui précèdent risquent de jeter un trouble, cependant. Entre l'évocation d'une pulpe du réel en tant que vécu, l'allusion à une totalité d'expérience plus vaste que celle du «monde extérieur », l'ontologie phénoménologique empruntée à Merleau-Ponty, et mon style prodigue en métaphores, on peut avoir l'impression d'entrer dans une atmosphère de pensée à peine moins spéculative que celle du matérialisme spéculatif. Cette grande ombre de représentation dans une approche qui se veut pourtant critique et transcendantale est la cible d'un réquisitoire convergent formulé par Isabelle Thomas-Fogiel et Anna Longo.

Anna Longo me reproche ce qu'elle nomme "les résidus métaphysiques impliqués par la phénoménologie», la référence à une "totalité vécue» qui serait la "réalité inconnaissable mais supposée comme fondement absolu ", le postulat de "la vérité absolue du fait de se savoir vivant». Elle se méfie aussi de mon usage d'une "épistémologie naturalisée» s'appuyant sur un critère de "satisfaction de l'utilité » dans le cadre d'un "jeu évolutionnaire ». La combinaison du spectre d'un fondement absolu, et d'un penchant vers la naturalisation de l'épistémologie, ne me reconduit-elle pas à une forme de «matérialisme »; celui-là même que j'avais voulu réfuter chez Meillassoux?

Isabelle Thomas-Fogiel pointe également des aspects fondationnalistes dans ma réflexion: "les contenus de l'endo-ontologie se révèleront le socle, la base, le sol de toute certitude. Toute action, réflexion, pensée, connaissance, deviendra relative au principe suprême: le "je vécu en son présent vivant" ". Elle s'étonne incidemment de mon choix d'un discours (fût-il détourné) sur l'être, en lieu et place d'une stricte adhésion au "vide de certains bouddhistes, qui implique un abandon de toute onto-logie, fût-elle d'immersion ». Et même si l'on abandonne l'endo-ontologie au profit d'un simple êtreimmergé, se demande-t-elle, pourquoi désirer ce dernier, "pourquoi vouloir l'immersion? ». De toute manière, le point d'appui de cette approche, simple "attestation muette d'un vécu singulier ", manque de la consistance voulue pour élaborer une ontologie à partir de lui. En phénoménologie, le constat vécu du vécu a représenté la "porte d'entrée et non (le) point d'arrivée » de 
l'enquête: porte d'entrée dans le domaine des invariants eidétiques de l'expérience, et non pas fin en soi paroxystique sous forme d'un auto-étant ultime. Pas plus qu'on ne trouve l'universel dans la nature, on ne peut le trouver dans le vécu, "car il n'est pas "donné" mais à réaliser".

Outre cette dérive ontologique sur la base de la phénoménologie, Isabelle Thomas-Fogiel dénonce une dérive épistémologique sur la base d'une science de la cognition. Cette dernière dérive est selon elle la craquelure qui mine l'ensemble de ma démarche. Il s'agit d'une option tacitement "darwinienne", selon laquelle la connaissance humaine est la pointe avancée de l'activité adaptative des êtres vivants. Avec pour conséquence, ici encore, que l'universalité est remplacée par l' "utilité », et que la vérité est remplacée par la "vraisemblance» localement efficace. Contre ce recours apparemment naiff aux leçons scientifiques du darwinisme et de l'énaction, Isabelle Thomas-Fogiel m'invite à leur appliquer la réduction phénoménologique, que je préconise partout ailleurs. Et contre ma tendance à rapatrier les questions philosophiques dans la singularité de la vie, tantôt la vie vécue en son unique présence, tantôt la vie biologique terrestre en son unique poussée historique, elle recommande d'ouvrir les deux multiplicités propres à la raison. La première est la multiplicité du temps des successions, qui nous permet de voir se déployer les contradictions performatives au lieu de les ressentir dans une commotion immédiate. "Pour établir la contradiction, souligne Isabelle Thomas-Fogiel, il faut admettre un léger différé entre mon dire "la vérité n'existe pas" et la conscience de mon faire qui invalide son contenu». La seconde multiplicité est celle des possibles, voire des mondes possibles, "par laquelle nous sortons à la fois du présent et de l'être », et qui par son usage du conditionnel «est peut-être le temps de la raison».

Il se confirmerait donc que ma réplique au matérialisme spéculatif suppose une spéculation tout aussi aventureuse, mais qui n'ose pas dire son nom? Une métaphysique inconsciente d'elle-même serait-elle bien la contrepartie de mes avertissements conscients contre la résurgence des illusions de la métaphysique?

Cette inquiétude n'est pas sans motifs, mais elle peut aisément être conjurée. Elle l'a d'ailleurs déjà été dans quelques phrases de Maintenant la finitude. "Ce qui est déclaré ne pas être dit n'est vraiment pas dit; ce qui est déclaré ne pas être pensé n'est vraiment pas pensé ${ }^{16}$.» La remarque est inspirée des derniers aphorismes du Tractatus logico-philosophicus. Malgré la trace des mots, Wittgenstein n'y dit vraiment pas que "le monde et la vie ne font qu'un ${ }^{17}$ ». Car il finit par retirer cette proposition, et les autres de ce genre, en indiquant que leur dire apparent n'avait d'autre but que d'inviter leur lecteur à voir pleinement ce qui, dans la vie, se présente comme monde.

16. Maintenant la finitude, p. 457.

17. L. Wittgenstein, Tractatus logico-philosophicus, Gallimard, 1993, \$5.621, p. 93 
Mais il faut à présent que je précise cette stratégie, ou que je m'en explique autrement.

Indubitablement, les substituts que j'ai proposés à l'ontologie de choses-devant-nous, et à la thèse d'un face-à-face entre le monde-sans-nous et le nous-sans-monde, ont tellement l'allure de représentations opposées à d'autres représentations, de récits endo-cosmiques visant à remplacer les grands récits cosmogoniques, qu'on peut se laisser captiver par leur sens manifeste. Pourtant, les représentations et les récits substitutifs que j'ai proposés n'ont, comme chez Wittgenstein, d'autre fonction que thérapeutique, à travers le procédé courant de l'antidote. Leur sens latent ne s'identifie pas à ce qu'ils semblent dire; il est celui d'un procédé de transformation du voir.

L'usage que je fais de l'endo-ontologie doit se comprendre de cette manière. Chez Merleau-Ponty, l'endo-ontologie s'éloigne déjà beaucoup de l'ontologie, puisqu'elle n'est pas un discours sur l'être, mais une tentative d'articuler discursivement la manifestation tacite de l'indifférenciation entre le locuteur et ce qui est. Elle est « une philosophie de l'inhérence, de l'habitation ${ }^{18} »$; elle n'est pas une philosophie de la déhiscence, de la caractérisation. Elle se reconnaît paradoxale, parce que, si elle utilise le procédé courant de la désignation par des noms, et de la prédication par des adjectifs, c'est pour signifier l'impossibilité de faire référence à autre chose que ce qui se vit et s'exprime durant le processus de désigner. Dans ces conditions, reconnaît Merleau-Ponty, le phénoménologue « devrait se taire, coïncider en silence, et rejoindre dans l'être une philosophie qui y est déjà faite ${ }^{19}{ }$. Le problème est qu'en écrivant cela Merleau-Ponty contrevient instantanément (et sciemment) à sa propre prescription. Car, en esquissant le mouvement de regagner son habitat originel, il fait comme s'il y avait là, devant son attention, un lieu d'être d'où il serait exilé et dont il se sait pourtant résident; il fait comme s'il y avait un quelque part comme «l'être» qu'il peut nommer dans sa position temporaire d'exil, avant d'oublier jusqu'à la sonorité de son nom lorsqu'il s'y est rapatrié.

Alors, plutôt que de rester enfermé dans ce type de contradiction, plutôt que d'avancer du bout des lèvres le concept d'inhérence propre à l'endo-ontologie tout en admettant son impropriété, il m'a semblé préférable d'aller jusqu'au bout de son discours, de le laisser se déployer assez longtemps pour qu'il remplisse son rôle d'antidote au discours ontologique. Une fois que l'endo-ontologie a pleinement déployé sa contre-image d'un réel insoustractible, une fois que cette contre-image a affaibli l'image "de bon sens » d'un réalisme soustractif, elle peut être écartée sans dommage, à

18. R. Decriem-Franksen, «L'apparaître à soi-même, ou l'émergence phénoménologique de la vie s'éprouvant elle-même comme continuité résistante ", sous la direction de M. Kronegger et AT. Tymieniecka in Life, Analecta Husserliana (The Yearbook of Phenomenological Research), 57, 175-199, Springer, 1998.

19. M. Merleau-Ponty, Le visible et l'invisible, Gallimard, 1964, p. 168. 
la façon de l'échelle de Wittgenstein. Mais pas avant. C'est seulement après avoir accompli son œuvre que le discours endo-ontologique peut être vu comme simple allégorie d'un geste de récollection post-discursif dans l'immanence de la vie vécue et pratique, ou comme introduction imagée à la sèche prescription du probabiliste radical d'Anna Longo: celle de parier et d'agir.

Il en va de même de mon usage d'expressions aussi chargées métaphysiquement que «l'expérience totale» ou le «je-ici-maintenant». Impliquentelles que la totalité vécue soit prise pour «fondement absolu», ou que "je vécu en son présent vivant " soit érigé en "principe suprême " ? La réponse à ces questions est négative, une fois encore. $\mathrm{Ni}$ fondement absolu, ni principe suprême, le présent vécu n'est poussé sur la scène des mots que pour signaler la possibilité de regagner son sûr refuge à chaque fois qu'un excès spéculatif nous égare, à chaque fois qu'un argument spéculatif semble nous déstabiliser. Autrement dit, les expressions «je-ici-maintenant» ou «l'expérience totale» ne désignent pas le socle inébranlable à partir duquel on serait en mesure d'avancer dans la connaissance, et sur lequel on pourrait construire avec sûreté l'édifice des principes et raisons du monde. Elles ne sont que l'allégorie d'un recul possible en ce lieu de coïncidence avec soimême que rien ne peut perturber parce qu'il fait sien toute perturbation. Loin de s'inscrire dans des actes de langage locutoires décrivant un pilier fondateur, les expressions «je-ici-maintenant» ou «l'expérience totale» n'ont pour vocation que de vivifier des actes de langage perlocutoires, ou plus précisément auto-locutoires ${ }^{20}$, visant à faire vivre ou à faire être (quelque chose à) quelqu'un.

Et qu'en est-il de l'immersion? Si j'ai manipulé cette image, ce n'est pas forcément par "goût " pour elle, mais là encore dans un but thérapeutique. Elle sert d'antidote évident à un projet multi-millénaire d'émersion, c'est-à-dire d'objectivation intégrale de ce qui est à savoir. Le projet d'objectivation ayant montré ses limites au cœur même des disciplines scientifiques qui en sont les pointes avancées, c'est seulement à condition de nous libérer de son pouvoir de fascination que nous comprendrons pleinement nos propres accomplissements théorétiques ${ }^{21}$.

Un exemple central d'usage de la fonction per-(ou auto-)locutoire des «mots de l'esprit» est offert dans l'argument que j'oppose à l'argument élenctique de Meillassoux. Il ne s'agit pas dans cet argument de nommer un acte de pensée présente, puis de l'ériger en preuve écrite (typique du cercle corrélationnel) que ce que l'on conçoit comme monde-indépendant-de-lapensée est encore pensé de ce seul fait. Il s'agit simplement de se servir d'expressions écrites comme «expérience présente» pour inviter l'interlocuteur

20. M. Bitbol, La conscience a-t-elle une origine?, Flammarion, 2014, p. 39.

21. M. Bitbol, "Making Sense of Non-Sense in Physics: the Quantum Koan », in M. Cappuccio \& T. Froese, Enactive Cognition at the Edge of Sense-Making, Palgrave-McMillan, 2014. 
qui formule la thèse du monde-non-pensé à s'apercevoir en silence qu'il la pense à l'instant où elle est formulée. L'instantanéité de l'aperception d'autocontradiction est ici cruciale. Car si on permet au matérialiste spéculatif d'échapper à la commotion instantanée de son auto-contradiction, de s'en souvenir et de la réfléchir, rien ne l'empêche de la transcrire en mots et en propositions, puis de l'empiler dans la série indéfinie des principes et métaprincipes de facticité qu'il prête à son adversaire corrélationniste et qu'il égrène pour mieux le piéger ${ }^{22}$.

C’est en cela que le procédé que j'appelle "contradiction existentielle» est vraiment distinct du procédé plus courant de "contradiction performative ». La contradiction existentielle doit sans cesse être réactivée pour faire sentir qu'elle est contraignante, tandis que la contradiction performative peut être constatée rétrospectivement, admise une bonne fois pour toute, et érigée en fondement (à la manière de Karl-Otto Apel, ou d'Aristote dans le livre Gamma de la Métaphysique).

Considérons l'exemple proposé par Isabelle Thomas-Fogiel: "pour établir la contradiction, il faut admettre un léger différé entre mon dire "la vérité n'existe pas" et la conscience de mon faire qui invalide son contenu: “je réalise que, disant cela, je présuppose qu'il y a au moins une vérité" ». Que se passe-t-il ici? Lorsque j'énonce "la vérité n'existe pas", je traite implicitement ma proposition comme relevant d'un méta-discours portant sur toutes les autres productions discursives; et je l'excepte donc de sa propre juridiction négative. Mais dire cela est encore trop abstrait, trop intemporel. Ce qui arrive ici concrètement est que je ne me perçois pas énonçant cette proposition qui porte sur toutes les autres propositions. Je ne me perçois pas énonçant pendant que j'énonce, pas plus que je ne me sais conscient pendant que j'ai conscience de quelque chose. Je suis sourd au parler du parlant, comme je suis aveugle au voir du voyant. Sans cette surdité à moi-même parlant, je ne pourrais pas adhérer un seul instant à ma proposition affirmant l'absence de propositions vraies. Puis, je me rends compte après-coup du fait que je viens d'émettre une proposition, et je confronte ce fait au contenu de la proposition émise. Rétrospectivement, je m'entends avoir parlé, je me comprends dans ce que je voulais dire. Je me rends compte que ma proposition antérieure revendiquait la vérité, et que je me suis donc contredit en affirmant son contenu. Une fois cette prise de conscience accomplie, mon auto-contradiction devient une certitude sur laquelle il ne sera plus question de revenir. Une fois cette prise de conscience accomplie, j'ai mis mon acte passé d'énonciation (mon faire) en contraste permanent avec le contenu de mon énoncé passé (mon dire).

C'est ce genre de contradiction performative que le «cercle corrélationnel» de Berkeley prétendait exhiber. La proposition «il y a une réalité indépendante de la pensée» (ou «telle est la réalité indépendante de la 
pensée ») étant rétrospectivement identifiée comme une pensée, un constat de contradiction performative devrait suffire à réfuter cette proposition une fois pour toutes. Mais Meillassoux a ouvert une brèche qui déstabilise cette conclusion, à chacune des étapes où elle est formulée ${ }^{23}$. Et il pousse le processus d'étape en étape, dans une régression que le corrélationniste ne parvient pas à fixer en une conclusion écrite qui lui serait favorable. Le seul moyen qu'a le corrélationniste d'emporter la décision face au matérialiste spéculatif est alors de saisir ce dernier au vol dans l'aboutissement de sa réfutation performative du corrélationnisme. Le seul moyen est d'inviter le matérialiste spéculatif à constater maintenant que lui pense avoir montré que quelque chose échappe à la corrélation avec la pensée. Le seul moyen est en d'autres termes de l'inviter à constater qu'il pense à l'instant cette chose supposée indépendante de la pensée (en l'occurrence le principe de factualité). L'imputation de contradiction performative (celle du «cercle corrélationnel ») ayant été mise en échec par celui qu'elle visait (le matérialiste), la seule option qui subsiste est de lui faire ressentir immédiatement la contradiction existentielle propre à sa position.

Aurais-je pu faire l'économie de ces antidotes? Aurais-je pu, par exemple, emprunter un raccourci intellectuel, en me prévalant d'emblée du "vide de certains bouddhistes, qui implique un abandon de toute onto-logie, fût-elle d'immersion? ». Ce serait oublier l'histoire de ce vide-là, qui n'est (éventuellement) atteint qu'à l'issue d'une longue cure dialectique des troppleins de la pensée. L'œuvre de Nâgârjuna, dans laquelle le concept de vacuité (Śñnyatā) tient la place centrale, se présente comme une séquence de renvois dos à dos de toutes les thèses de type ontologique. Chaque thèse, depuis le réalisme chosiste des atomistes indiens, jusqu'à l'idéalisme hérité des Upanishads, est repoussée après l'autre. Mais, dans le cursus d'enseignement qui s'appuie sur cette œuvre, la dissolution finale n'est présentée qu'après que chaque thèse $\mathrm{a}$ eu le temps de corroder la précédente. La mise hors-jeu de toutes les thèses a ainsi temporairement besoin de la thèse idéaliste, afin de faire voir la thèse du réalisme chosiste comme l'illusoire projection de nos catégories verbales et mentales sur une réalité "extérieure ". Aucune thèse n'est retenue en fin de parcours, mais chaque thèse a eu la possibilité d'opérer sur un mode thérapeutique à mi-parcours. Puis, aucune caractérisation ontologique n'ayant été retenue, chaque entité ayant été trouvée "vide » d'être-propre, même le concept de "vacuité » est emporté dans le flot. Le mot "vacuité " avait simplement pour fonction d'attirer l'attention du lecteur vers l'arasement mental que la critique de l'ontologie est capable d'accomplir. Il avait simplement pour but de recueillir réflexivement ceux qui le comprennent dans la fraîcheur stupéfaite d'un regard dont les

23. Il la déstabilise en montrant qu'à chaque fois que le corrélationniste la prononce, il oppose ce qui est pensable par nous à ce qui serait (mais ne peut pas être) pensable comme «en soi ». 
cadres conceptuels, et les formes pré-conceptuelles de la perception, viennent d'éclater sous la poussée d'une critique illimitée ${ }^{24}$.

C'est ainsi que je comprends également l'usage des gestes fondateurs de la phénoménologie. J'accorde à Isabelle Thomas-Fogiel que, dans le corpus central de l'œuvre de Husserl, le geste suspensif de l'épochè et le geste réflexif de la réduction servent de "porte d'entrée et non (de) point d'arrivée". J'accorde que l'ordre méthodique de la phénoménologie suppose l'antériorité de l'acte double d'épochè et de réduction, et la postériorité du fruit de la recherche qu'est la circonscription des "essences» des vécus. Mais qu'en est-il de l'ordre des motivations? Husserl insiste trop souvent (dans la Philosophie première et dans la Krisis) sur la signification éthique, et sur le pouvoir décisif d'auto-transformation de l'épochè pour que cette dernière n'ait été pour lui, pour sa personne singulière en deçà de sa persona philosophique, qu'un moyen existentiel en vue d'une fin épistémique. Aurais-je besoin d'un "complet changement de l'attitude de l'ensemble de la vie, un mode du vivre totalement nouveau ${ }^{25}$ ", si c'était seulement pour accéder, dans un but descriptif, aux structures de la "conscience pure»? Devrais-je rendre permanent le "point de vue transcendantal ", et le laisser «déterminer en nécessité toute la praxis de ma vie »"26, si ce n'était que pour m'adonner transitoirement à l'activité professionnelle de la description phénoménologique des invariants eidétiques du flux de la conscience? Cela paraît si peu plausible qu'on a pu proposer une complète inversion de l'ordre des priorités motivationnelles chez Husserl ${ }^{27}$. Le long et scrupuleux exercice des réductions et des descriptions eidétiques ne serait au fond qu'une forme laïcisée d'exercice spirituel, qu'un prétexte et une propédeutique pour accomplir puis pour pérenniser l'état d'épochè. Ce qui rend cette inversion crédible n'est certes pas l'ensemble des contenus explicites de l'œuvre husserlienne, tendus vers le projet d'une "science rigoureuse» des essences vécues. Ce qui rend l'inversion crédible, c'est la vie même d'Edmund Husserl; sa vie dont il a fait une durable ascèse, que le simple désir de mettre au jour l'univers jusque-là inexploré de la conscience pure n'aurait sans doute pas suffi à justifier.

Les relents de métaphysique qui peuvent affleurer ici et là dans Maintenant la finitude, y compris dans ses thèmes phénoménologiques, viennent d'être reconduits à leur fonctionnement auto-locutoire et à leur finalité thérapeutique. Qu'en est-il à présent des passages qui relèvent d'une théorie de la connaissance naturalisée; d'une conception adaptative, darwi-

24. M. Bitbol, «Neurophenomenology of Surprise», in N. Depraz \& A. Celle, Surprise at the Intersection of Phenomenology and Linguistics, John Benjamins, 2019.

25. E. Husserl, La crise des sciences européennes et la phénoménologie transcendantale, Gallimard, 1976, p. 171.

26. Ibid., p. 113.

27. Je renvoie sur tous ces points à l'excellente thèse soutenue le 14 mai 2020 à l'Université de Montréal par Philippe Blouin sous le titre La phénoménologie comme manière de vivre. 
nienne, ou énactive des théories scientifiques? Sont-ils le signe d'une régression pré-philosophique, d'un affaiblissement de la posture transcendantale, d'une retombée dans l'attitude naturelle? Je pense m'en être expliqué dans la partie III de De l'intérieur du monde. En bref, une épistémologie naturalisée n'est pas proposée en lieu et place d'une épistémologie transcendantale. Elle l'est à titre de terrain d'épreuve figuratif "permettant de s'assurer que l'épistémologie (transcendantale) n'entre pas en contradiction avec le produit d'une science élaborée selon ses propres prescriptions ${ }^{28}{ }^{2}$. Entre les normes transcendantales et les inévitables tentatives de représenter, conformément à ces normes, "la genèse du transcendantal dans le champ naturel ${ }^{29}$ ", un cercle d'auto-consistance doit s'établir. Une bonne épistémologie naturalisée a pour première mission de tester itérativement ce cercle d'autoconsistance, jusqu'à s'y inscrire de manière harmonieuse.

La deuxième mission que j'assigne à une théorie de la connaissance naturalisée, comme le rappelle Isabelle Thomas-Fogiel, est de mobiliser la pensée gnoséologique. La théorie de la connaissance a vraiment besoin d'utiliser tour à tour ses deux points d'appui, naturaliste et transcendantal. Elle en a besoin pour avancer, pour ne pas figer les normes déduites régressivement (sur le mode de la déduction transcendantale kantienne) à partir d'un état donné de la connaissance qu'elles ont rendu possible. "Se figurer la relation cognitive comme si elle était elle-même un objet de connaissance permet de mettre sa particularité au jour et d'imaginer la manière dont elle pourrait évoluer vers d'autres formes particulières afin de donner lieu à de nouveaux cycles de recherches stabilisées ${ }^{30}$.»

Il faut remarquer par ailleurs que, dans une perspective darwinienne ou énactive, nulle théorie naturalisée de la connaissance ne saurait prétendre être (ou approcher) une représentation fidèle de ce qu'est la connaissance. Selon ces théories naturalisées de la connaissance, en effet, aucune théorie scientifique ne peut être considérée comme une représentation fidèle (ou tendant vers la fidélité) de ce qui est dans le monde. Or, en tant que théories scientifiques de la connaissance, elles tombent sous le coup de leur propre verdict. En appliquant réflexivement leur enseignement à elles-mêmes, on doit les considérer non pas comme des représentations, mais comme des opérateurs plurivalents. Selon les perspectives darwinienne ou énactive, les théories scientifiques standard sont des opérateurs dans l'économie de la connaissance. Elles permettent de poser les paradigmes d'action et d'énonciation dans le cadre desquels vont se dérouler de longues séquences historiques de recherche. Selon la même perspective, les théories scientifiques (naturalisées) de la cognition sont pour leur part des opérateurs dans l'économie d'une connaissance de la connaissance. Elles servent d'échafaudages

28. M. Bitbol, De l'intérieur du monde, Flammarion, 2010, p. 554.

29. Maintenant la finitude, p. 277.

30. Maintenant la finitude, p. 280. 
métaphoriques pour élaborer, par-delà un paradigme scientifique, la théorie normative et transcendantale de la connaissance qui le stabilise et en rend raison. Une première ébauche de théorie naturalisée de la connaissance a ainsi pu servir d'échafaudage métaphorique à l'élaboration de la théorie transcendantale de la connaissance adaptée au paradigme newtonien ${ }^{31}$; puis d'autres théories naturalisées de la connaissance sont venues offrir leur armature provisoire pour refonder une théorie transcendantale de la connaissance procédant comme norme rectrice et condition de possibilité pour de nouveaux paradigmes ${ }^{32}$, et ainsi de suite.

Toutefois, cette fonction d'opérateur architectonique dans la dynamique d'édification des strates de la connaissance n'est pas la seule que l'on puisse attribuer aux théories naturalisées de la connaissance. Comme les esquisses métaphysiques, elles servent aussi d'opérateur thérapeutique. Elles sapent le naturalisme à sa base naturelle. Car elles permettent de confronter les diverses formes du "réalisme scientifique » à leur incompatibilité avec les plus avancées et les plus crédibles parmi les théories scientifiques de la cognition. Comment la thèse d'une représentation de la réalité à connaître par les théories scientifiques peut-elle s'accorder avec les théories scientifiques qui font de la cognition le fruit d'une co-adaptation plutôt que d'un recueil unidirectionnel ? Comment la thèse d'une représentation de la réalité «indépendante » par le cerveau peut-elle s'accorder avec une neurobiologie qui enseigne que l'état de veille cérébrale équivaut à une activité onirique modulée à la marge par des états sensoriels co-émergents ${ }^{33}$ ? Husserl lui-même a utilisé le procédé de la contradiction réflexive contre le naturalisme (et le psychologisme) de son temps. Dans l'ambiance d'un naturalisme gnoséologique mettant en jeu "la théorie moderne de l'évolution ${ }^{34}$ », remarque-t-il, la certitude de la connaissance scientifique de la nature dont le naturalisme se prévaut est minée par ses propres conclusions.

Après le naturalisme, le matérialisme spéculatif peut aisément devenir la cible de ce genre de critique interne. L'extraordinaire audace du matérialiste spéculatif ne serait-elle pas contrainte par un extraordinaire conservatisme dans le champ de la théorie de la connaissance ? Parler de connaître un monde-sans-nous, cela ne suppose-t-il pas qu'on ait restreint implicitement l'épistémologie au modèle suranné de la connaissance spéculaire au sens de Rorty (nous comme miroir d'un monde qui nous fait face et nous exclut), ou au modèle de la commande robotique (alternant les entrées sensorielles et les

31. Maintenant la finitude, p. 278. Il s'agit bien entendu de la théorie transcendentale kantienne de la connaissance.

32. M. Bitbol, "Physique quantique et cognition ", Revue internationale de philosophie, 212, 299-328, 2000.

33. R.R. Llinas \& D. Paré, "Of Dreaming and Wakefulness ", Neuroscience, 44, 521$535,1991$.

34. E. Husserl, L'idée de la phénoménologie, Presses Universitaires de France, 1997, p. 42 . 
sorties motrices en provenance et vers le monde extérieur ${ }^{35}$ ? Si la connaissance scientifique du prétendu «monde sans nous» en arrive à la conclusion que le «nous» objectivé est structurellement indissociable du monde-objet, alors la tentative de nous soustraire du monde est invalidée par son propre aboutissement. Une fois ce retournement du réalisme scientifique contre luimême mis en évidence, il est temps de mettre à bas l'échafaudage de représentations cognitives (darwiniennes et énactives) qui l'a permis; il est temps de «jeter l'échelle (naturalisante) après y être monté ${ }^{36}$ ».

Une interrogation peut cependant naître du fait que je mets très (trop?) souvent en jeu une conception pour ainsi dire bergsonienne de la connaissance théorique ( «nous tenons l'intelligence humaine pour relative aux nécessités de l'action $\left.{ }^{37} »\right)$. L'objectif premier de cette conception est d'affaiblir les prétentions hyperboliques de la raison dans le matérialisme spéculatif, de leur opposer, sur un mode réflexif, le produit même de la raison scientifique. Mais l'accumulation des citations de Maintenant la finitude produites par Isabelle Thomas-Fogiel fait ressortir une telle prégnance du modèle biologique de la connaissance, qu'on peut légitimement se demander s'il n'y est pas pris plus au sérieux qu'un simple antidote.

Je reconnais que c'est le cas; que les modèles darwinien et énactif me servent constamment (et non pas ponctuellement, comme remède transitoire) de point d'appui lorsque je cherche à m'orienter dans le champ de la théorie de la connaissance. Mais ce recours à une théorie naturalisée de la connaissance n'est pas exclusif, et il ne se prévaut pas de la seule autorité des sciences de la nature. Je ne considère la théorie naturalisée de la connaissance comme crédible, une fois de plus, que dans la mesure où elle se coule harmonieusement dans un cercle d'auto-consistance qui inclut, en plus d'elle-même, une théorie transcendantale de la connaissance, et une évaluation interprétative du mode de fonctionnement des théories scientifiques les plus avancées. Ainsi, que le jeu des anticipations et des confirmations ou infirmations sensorielles soit le soubassement de la connaissance n'est pas affirmé au seul nom d'une théorie biologique de la cognition. Cette affirmation est rendue systémiquement crédible par la relation d'isomorphisme qui unit une certaine théorie naturalisée de la connaissance à une théorie transcendantale de la connaissance, et à une interprétation clarifiante de la mécanique quantique. Aux anticipations motrices des êtres vivants (1), répondent les horizons d'attentes qui participent de l' «essence» phénoménologique de la perception (2), et les paris réglés en quoi consistent les probabilités quantiques selon le QBism (3). Aux confirmations / infirma-

35. Pour une critique de cette conception de l' ' intelligence» des robots, voir B.C. Smith, On the Origin of Objects, MIT Press, 1998; B.C. Smith, The Promise of Artificial Intelligence, MIT Press, 2019.

36. L. Wittgenstein, Tractatus logico-philosophicus, op. cit. $\$ 6.54$.

37. H. Bergson, L'évolution créatrice, Presses Universitaires de France, 2018, p. 153. 
tions sensorielles (1), répondent le remplissement ou la déception des attentes (ainsi que leur réorientation) en phénoménologie (2), et l'attestation expérimentale qui impose d'ajuster les paris réglés en mécanique quantique (3). Il en résulte que l'enseignement que je tire des théories naturalisées darwinienne et énactive de la connaissance pourrait aussi bien se prévaloir de l'un des deux autres éléments, transcendantal et quantique, du cercle d'auto-consistance dans lequel s'insèrent ces théories. Mais il n'est suffisamment justifié que par l'adéquation réciproque des trois éléments du cercle; et il est mieux illustré par l'image naturalisante que par ses deux répondants non imagés.

Les trois éléments précédents du cercle d'auto-consistance épistémologique convergent aussi pour suggérer que l'idéal d'accord universel n'a pas à être poursuivi sur le mode d'une quête de vérité-correspondance, mais sur deux modes alternatifs. L'idéal d'accord universel opère d'une part sur le mode de la recherche consensuelle d'optimum performatif (sciences de la nature), et d'autre part sur le mode de la tension personnelle et collective vers la cohérence interne d'un système symbolique (mathématiques). Admettons de surcroît (dans l'esprit des philosophies conventionnaliste ${ }^{38}$, intuitionniste, pragmatiste, piagétienne ${ }^{39}$, ou wittgensteinienne ${ }^{40}$ des mathématiques) que les symboles entre lesquels la cohérence interne est imposée n'expriment rien d'autre que des ensembles réglés de possibilités performatives ouvertes aux êtres situés que nous sommes. Alors, on voit immédiatement comment peuvent entrer en synergie, au lieu de s'opposer, la contrainte instantanée de l'action et le «temps de la raison» qu'est le possible.

Il me reste à aborder le thème du présent et de la présence, que Claudia Serban considère à juste titre comme la clé de voûte de ma réponse au matérialisme spéculatif. La thèse que l'ultime relativité épistémique, par laquelle nous constituons le cosmos jusqu'en son passé ancestral, n'est autre que la relativité au présent vivant; la thèse que ce dont les objets et événements sont des corrélats intentionnels est l'acte d'une conscience actuelle. Elle seule permet de maintenir que tous les objets et les événements de l'univers sont des corrélats d'un vécu intentionnel de conscience, contre la forte objection de l'absence d'êtres humains dotés de conscience dans le passé de l'univers. Ce point de repli ultime du corrélationnisme est bien identifié par Meillassoux; mais il est sérieusement mésinterprété par lui. Selon ses propres termes, il revient à accepter que le "sens authentique» de l'antériorité des événements «ancestraux» est de "n'être qu'une rétrojection par la pensée

38. P. Narbonnand, «La théorie de l'espace de Poincaré », sous la direction de P. E. Bour et S. Roux, in Lambertiana, Recherches sur la philosophie et le langage, Vrin, 2010.

39. J. Piaget, Introduction à l'épistémologie génétique I: la pensée mathématique, Presses Universitaires de France, 1972.

40. J. Bouveresse, Le pays des possibles, Éditions de Minuit, 1988. 
présente d'un passé se donnant à la pensée comme antérieur à la pensée ${ }^{41}$ ». Une telle option "présentiste» est tournée en dérision par Meillassoux pour deux raisons dont la formulation révèle deux incompréhensions. La première raison est que "relatif au présent " équivaudrait, ou peu s'en faut, à affirmer que seul le présent existe ${ }^{42}$; or, comme le souligne Claudia Serban, la thèse de la relativité (au présent) n'implique pas la thèse de l'existence (du seul présent). La seconde raison dont se prévaut Meillassoux est l'absurdité alléguée d'une «rétrojection» du "passé anté-humain " «à partir d'un présent humain lui-même historiquement situé ${ }^{43}$; or, dans un cadre phénoménologique, la relativité des événements passés au présent vivant est clairement distincte de leur relativité à un instant empiriquement localisé du temps historique humain.

Une fois ces deux incompréhensions dissipées, ma charge contre l'anticorrélationnisme du matérialiste spéculatif se poursuit, en se prévalant d'une conception du rapport entre sciences et philosophie qui diffère profondément de la sienne. Pour le matérialiste spéculatif, le philosophe a pour tâche de justifier le sens obvie, littéral, des énoncés par lesquels les chercheurs scientifiques communiquent leurs résultats, tout particulièrement leurs conclusions cosmogoniques ou paléontologiques. Pour le corrélationniste, il s'agit au contraire de creuser sous la couche du sens obvie pour mettre au jour une couche de sens plus profonde de l'œuvre scientifique, souvent ignorée des chercheurs eux-mêmes. Cela, non pas pour leur enseigner un «modeste savoir» philosophique ${ }^{44}$ dont ils n'ont que faire, mais pour les aider à surmonter leurs propres crises des fondements au moyen d'un geste réflexif. L'exemple de la levée de quelques soi-disant "paradoxes" quantiques par une approche corrélationniste radicale illustre cette conception alternative du rapport entre sciences et philosophie ${ }^{45}$.

Avant de poursuivre l'examen des objections de Claudian Serban, revenons brièvement à la question délicate de savoir si j'attribue une existence exclusive au présent, à laquelle serait opposée l'inexistence de toutes les phases du passé aussi bien que du futur. La réponse est négative. Si les choses apparaissent (et sont donc) maintenant, si les trois phases du temps sont constituées maintenant, alors maintenant lui-même ne peut être ni un étant, ni un temps. Mais comme maintenant ne saurait être rien d'autre qu'un étant ou un temps, on ne peut même pas dire qu'il est. Ce que Hegel nomme «le maintenant qui est ${ }^{46}{ }^{»}$, celui que l'on peut considérer comme le

41. Q. Meillassoux, Maintenant la finitude, Éditions du Seuil, 2006, p. 169.

42. Ibid., p. 36. Le corrélationniste est ici rapproché du créationniste qui pense que les traces fossiles évoquant un lointain passé évolutif ont été engendrées par Dieu en un seul jour récent. Pour une réponse à cette comparaison, voir Maintenant la finitude, p. 435 et suiv.

43. Ibid., p. 170.

44. Ibid.

45. Maintenant la finitude, p. 408 et suiv.

46. G.W.F. Hegel, La phénoménologie de l'esprit, Aubier, 1999, p. 88. 
maintenant effectif parce qu'il n'a pas encore été réfléchi et n'a donc pas encore été rejeté dans le passé, doit au contraire être compris comme maintenant qui n'est pas. Et ce que Hegel nomme «le maintenant qui n'est pas ", celui qui vient d'être réfléchi et n'est donc déjà plus maintenant, doit au contraire être compris comme un instant particulier qui est: un instant ponctuel posé là devant notre attention, en tant qu'objet de réflexion et de représentation chronométrique. "Maintenant » désigne ce à quoi tout ce qui se présente est relatif, sans être lui-même présenté, ni comme chose ni comme instant.

Mais alors, qu'est-ce qui se présente, et, à partir de là, qu'est-ce qui est? Ce qui se présente, et partant ce qui est, n'est autre que le contenu entier du présent vivant, c'est-à-dire son passé immédiat en rétention. Sur ce point, j'approuve Claudia Serban, qui signale que notre présent enveloppe «l'intervalle ou la continuité entre le maintenant et le tout-juste-passé qui est encore présent». Je m'accorde encore avec elle, lorsqu'elle récuse toute symétrie entre le statut phénoménologique du passé (présent en chair et en os, au moins partiellement, sur le mode rétentionnel), et celui du futur dont la seule présence est celle, schématique, des projets et anticipations qu'il suscite. Mais j'insisterai plus fortement qu'elle sur le fait que, dès qu'on n'est plus en prise avec le tout-juste-passé rétentionnel, l'accès au passé se fait précaire. Au mieux, nous avons affaire à des souvenirs personnels, voire à une pleine résurrection ${ }^{47}$ de scènes passées (à la manière de la madeleine de Proust). Au pire, nous nous contentons de témoignages, de documents, de traces, d'archives. Mais dans ce dernier cas, l'accès au passé dépend de conjectures soumises à des réfutations possibles, c'est-à-dire d'un futur de recherche des traces encore inconnues.

D'un mode d'accès à l'autre, le coefficient de certitude du passé le plus immédiat est transféré de proche en proche au plus médiat. Nous nous souvenons d'une mélodie, mais nous sommes (presque) certains de l'avoir entendue en concert, parce que nous nous souvenons aussi qu'elle a été vécue par nous comme tout-juste-passée dans la traînée rétentionnelle laissée par l'orchestre. Nous voyons une photographie, mais nous sommes (presque) certains qu'elle renvoie à notre propre passé parce que nous pouvons la rapporter à un souvenir qui vient d'être éveillé par elle. Nous lisons une archive historique, mais nous sommes (presque) certains qu'elle renvoie au passé d'autres êtres humains, parce qu'elle équivaut aux journaux intimes qui témoignent de notre propre passé. Nous mettons une trace au jour, strate archéologique ou reliquat d'un impact météoritique, mais nous sommes (presque) certains qu'elle renvoie au passé de choses non humaines, parce que des annales humaines font allusion à un événement (historique, astronomique) observé. Et ainsi de suite, jusqu'à l'archi-fossile de Meillassoux. Lui ne peut même pas bénéficier de la concordance avec une annale humaine, et

47. G. Gusdorf, Mémoire et personne, Presses Universitaires de France, 1950, p. 68. 
son sens est donc suspendu à un développement futur de la quête paléontologique ou cosmologique.

Il faut se garder de toute fascination pour cette cascade de transferts de quasi-certitude par contiguité. Dans le cadre d'une attitude phénoménologique, un tel mode de transfert doit également être réduit. Et la réduction doit s'exercer dès la première étape, celle du passage de la rétention au souvenir. "Un souvenir, écrit Husserl, me donne accès au transcendantal de deux manières. [...] D'une part, le “je me souviens" m'est à présent donné dans le cadre de ma vie transcendantale actuelle; et d'autre part, ce "je me souviens" évoque ma vie transcendantale passée ${ }^{48}$." La vie transcendantale passée n'est présente que par évocation, à la différence de la vie transcendantale actuelle qui est présente tout court. Husserl utilise dès lors deux mots distincts pour ces deux modes de présence: "présentation» (pour le tout-juste-passé retenu en présence), et "présentification " ${ }^{49}$ (pour le passé évoqué). Il en résulte que «le passé [...] n'est que comme passé du présent ${ }^{50}$ »; il est tantôt présenté, tantôt présentifié. De là à conclure que «l'absolu luimême est ce présent originaire universel; en lui réside tout temps et tout monde ${ }^{51}$ ", il n'y a qu'un pas auquel Husserl ne s'est pas dérobé.

C'est en franchissant ce pas que Husserl a pu répondre de manière décisive au soupçon de solipsisme transcendantal que suscite le procédé de constitution de l'alter ego à partir de l'ego, dans les Méditations cartésiennes. C'est en accédant à une couche pour ainsi dire pré-egotique de l'expérience qu'il a pu remonter en deçà de la réduction cartésienne. Cette couche originaire, c'est celle d'un «je » vide d'identification à un soi biographique, vide d'adhésion à des manières d'être personnelles, vide même de souci de soi. Un pur présent qui n'est indiqué par le pronom personnel «Je" qu'à mesure de son caractère d'ouverture située, et non pas (contrairement à la conception du paragraphe 32 des Méditations cartésiennes) en raison de son rattachement au sillage des habitus individuels.

Cette couche originaire, c'est celle qu'a perçue Rousseau à l'issue d'un accident, et qu'il décrit dans la deuxième des Rêveries d'un promeneur solitaire: «Tout entier au moment présent je ne me souvenais de rien; je n'avais nulle notion distincte de mon individu, pas la moindre idée de ce qui venait de m'arriver; je ne savais ni qui j'étais ni où j'étais; je ne sentais ni mal, ni crainte, ni inquiétude. » Je non distinct de tu et de il $y$ a: tel est l'universel concret qu'on réintègre quand on a suspendu (volontairement ou accidentel-

48. E. Husserl, Philosophie première 2, Presses Universitaires de France, 1972, p. 121.

49. Le mot "présentification" traduit le mot allemand «Vergegenwärtigung " qu'utilise Husserl.

50. E. Husserl, «Réduction au présent vivant comme sol de toutes mes validités », Epokhè, nº 2, Jérôme Millon, 1991, p. 379.

51. Hua XV, n 38, p. 668, traduit, cité et commenté par N. Depraz, «Temporalisation de l'absolu selon Husserl », Epokhè, n 2, Jérôme Millon, 1991, p. 399; E. Husserl, Philosophie première 2, op. cit. p. 241. 
lement) l'effort vers un universel abstrait. Se pourrait-il à l'inverse que la poussée vers l'idéal d'un universel abstrait soit le déploiement dans le temps de cet universel concret toujours-déjà disponible au présent de l'indicatif ? 